\title{
Fungos micorrízicos arbusculares em sistema de aléias no Estado do Maranhão, Brasil
}

\author{
Camila Pinheiro NOBRE ${ }^{1}$, Altamiro Souza de Lima FERRAZ JÚNIOR², Bruno Tomio GOTO³ , Ricardo Luís \\ Louro BERBARA ${ }^{4}$, Marcelo Diógenes Costa NOGUEIRA ${ }^{5}$
}

\begin{abstract}
RESUMO
Sistemas em aléias podem consistir numa solução para o uso da terra em regiôes do Trópico Úmido. A relação dessa forma de manejo com a dinâmica dos fungos micorrízicos arbusculares (FMA) ainda é pouco compreendida. O objetivo desse estudo foi verificar a influência de leguminosas arbóreas em um sistema em aléias na capacidade infectiva e diversidade de FMA nativos em São Luís, Maranhão. Amostras de solo coletadas do sistema em aléias no campus experimental da Universidade Estadual do Maranhão (UEMA) - São Luís, em duas épocas do ano (Julho/2006 e Abril/2007), a duas distâncias (0 m e 0,5 m) do tronco de três leguminosas (Leucaena leucocephala, Clitoria fairchildiana e Acacia mangium) e em área testemunha (sem leguminosas) na profundidade de $0-20 \mathrm{~cm}$. O solo coletado foi utilizado para avaliar a capacidade infectiva dos FMA nativos, densidade e identificação de glomerosporos. O sistema em aléias aumenta o potencial de infectividade dos FMA nativos dependendo da espécie de leguminosa arbórea associada, estação de coleta e proximidades das árvores. Dezesseis espécies de FMA foram encontradas na área distribuídas em cinco gêneros, sendo Scutellospora o mais representativo.
\end{abstract}

PALAVRAS-CHAVE: Sistema Agroflorestal; Diversidade; Micorriza;

\section{Arbuscular mycorrhizal fungi in an alley cropping system in the state of Maranhão, Brazil}

\begin{abstract}
Alley cropping systems may be a solution for land use in tropical regions. How land use is connected to mycorrhizal arbuscular fungi (AMF) is poorly understood, especially in the tropics. The aim of this study was to evaluate the influence of leguminous trees in an alley cropping system in regard to the infectivity and diversity of native AMF species in São Luís, Maranhão, Brazil. Soil samples were collected in an experimental area of the Universidade Estadual do Maranhão (UEMA) - São Luís, in two seasons (July/2006 and April/2007), two distances $(0 \mathrm{~m}$ and $0.5 \mathrm{~m}$ ) from the trunk of three leguminous trees (Leucaena leucocephala, Clitoria fairchildiana e Acacia mangium), and in a control area 0-20 cm deep. Soil samples were used to evaluate the infectivity of AMF, density and diversity of glomerospores. The alley cropping systems increased the infectivity of AMF which varied according to the leguminous tree species, raining season and distance from trees. Sixteen AMF species of five genera were identified in the area, and Scutellospora was the most representative genus.
\end{abstract}

KEYWORDS: Agroforesty system; Diversity; Mycorrhiza;

\footnotetext{
1 Universidade Federal Rural do Rio de Janeiro; Email: camilaenobre@yahoo.com.br

2 Universidade Estadual do Maranhão; Email: aferrazjr@yahoo.com

3 Universidade Federal do Rio Grande de Norte; Email: brunogoto@hotmail.com

${ }^{4}$ Universidade Federal Rural do Rio de Janeiro; Email: berbara@ufrrj.br

${ }^{5}$ Universidade Estadual do Maranhão; Email: marcdiogenes@hotmail.com
} 


\section{INTRODUÇÃO}

Os solos tropicais são frágeis devido aos efeitos de tempo e clima, podendo ser degradados com maior facilidade, restringindo sua utilização para a produçáo de alimentos (Cardoso e Kuyper 2006). Tendo como objetivo melhorar a capacidade produtiva desses solos foi proposto um modelo de agricultura que mimetizasse uma floresta, denominado sistema agroflorestal (SAF). Esse sistema pode aumentar a disponibilidade de nutrientes e acelerar a ciclagem do fósforo (P) devido à presença de raízes em camadas mais profundas do solo; melhorar a qualidade química e física, assim como a atividade microbiana dos mesmos (Cooper et al. 1996).

Localizado no chamado Trópico Úmido, o Estado do Maranhão possui solos com baixa fertilidade. Sua agricultura característica é do tipo itinerante ou corte-queima, a qual consiste no uso da terra alternando períodos curtos de pousio e cultivo (Ferraz Júnior 2006). Uma opção para substituir a agricultura do tipo corte e queima, é o cultivo em aléias, um dos sistemas agroflorestais mais simples que combina espécies arbóreas, preferencialmente leguminosas, e culturas anuais ou perenes de interesse econômico em uma mesma área (Sanchez 1995). Essa prática garante ao solo cobertura morta, reduz o processo erosivo, permite a formação de microclima e retenção de umidade, que favorece o desenvolvimento de microrganismos benéficos às plantas (Cooper et al.1996). Entre as interaçóes de complementaridade se destacam: o suprimento de nutrientes na zona radicular das culturas por meio da entrada de $\mathrm{N}_{2}$ por fixação biológica, redução das perdas de nutrientes por lixiviação devido à absorção em camadas mais profundas e reciclagem de resíduos orgânicos provenientes da deposição de serapilheira ou, ainda, incorporação de biomassa por meio da adubação-verde (Buresh e Tian 1998; Rowe e Cadish 2002; Bala et al. 2003).

Os benefícios do sistema em aléias para a microbiota do solo podem influenciar a dinâmica dos fungos micorrízicos arbusculares (FMA). Estes organismos pertencentes ao filo Glomeromycota (Schüßler et al. 2001), com atualmente, 19 gêneros conhecidos, formam simbiose com a maioria das famílias de angiospermas, sendo comum em todos os tipos de ecossistemas terrestres: desde as regióes polares até florestas tropicais (Read 1991; Nielsen et al. 2004).

Poucos estudos sobre a dinâmica de FMA em SAF são conhecidos. Diagne et al. (2001) avaliaram o potencial de infectividade dos fungos micorrízicos (MIP) em sistema em aléias com três espécies de leguminosas (Acacia nilotica (Schum.), A. tortilis (Forsk) Hayne e Prosopis juliflora (Swartz) D.C.) no Senegal e verificaram que a concentração das raízes e de glomerosporos são bons indicadores de MIP e que há um potencial benefício dos sistemas em aléias devido à manutenção de alta concentração de inóculo na área. Pande e Tarafdar (2004) verificaram a ocorrência predominante de
Glomus e Gigaspora em SAF com neem (Azadirachta indica L.) em Rajasthan, Índia. Oyetunji e Osonubi (2007), na Nigéria, verificaram que o cultivo de mandioca inoculada com fungos micorrízicos em sistemas em aléias é mais produtivo devido à constante multiplicação dos FMA e aos benefícios nutricionais das leguminosas. No Brasil, estudos com FMA e SAF têm se concentrado na cultura do cafeeiro (Coffea arabica L.). Cardoso et al.(2003) verificaram a distribuição espacial dos glomerosporos e verificaram que quanto maior a profundidade, menor a densidade desses propágulos. Bonfin et al. (2010) verificaram a influência do sombreamento e da disponibilidade hídrica em cafeeiros consorciados ou não com grevíleas (Grevillea robusta A. Cunn.) e observaram maiores números de glomerosporos e maior taxa de colonização radicular nas áreas sombreadas.

Os estudos de sistemas em aléias relacionados com fungos micorrízicos arbusculares são escassos, principalmente na dinâmica sazonal desses organismos. Este trabalho tem como principais objetivos verificar a densidade de glomerosporo, a capacidade infectiva e a diversidade de FMA nativos de um sistema em aléias no município de São Luís, Maranhão.

\section{MATERIAL E MÉTODOS}

O sistema em aléias estudados se localiza no Campus da Universidade Estadual do Maranhão (20 35'39" Latitude Sul e 440 12'42" Longitude Oeste) e foi instalado em janeiro de 2002. Para o presente estudo foram selecionadas as três espécies de leguminosas com melhor estabelecimento no campo experimental (acácia - Acacia mangium Willd., leucena - Leucaena leucocephala (Lam.) R. de Wit e sombreiro - Clitoria fairchildiana R. Howard). O experimento foi implantado em quatro blocos ao acaso com área de $500 \mathrm{~m}^{2}$ cada. Amostras de solo foram coletadas na proximidade de quatro árvores de cada espécie, em cada bloco, evitando-se as árvores localizadas nas extremidades das linhas, totalizando-se 16 árvores amostradas de cada espécie. As coletas foram realizadas em duas estaçóes do ano: na época seca (julho/2006) e na época chuvosa (abril/2007) na profundidade $0-20 \mathrm{~cm}$ a duas distâncias do tronco das árvores $(0 \mathrm{~m}$ e $0,5 \mathrm{~m})$.

As amostras de solo coletadas foram acondicionadas em sacos plásticos, levadas ao laboratório e armazenadas em geladeira até determinaçóes de número de glomerosporos e realização de bioensaio. Para análise dos parâmetros químicos de rotina de fertilidade foram homogeneizados os solos coletados das mesmas leguminosas nos quatro blocos. De uma parte do solo, realizou-se peneiramento úmido (Gerdemann e Nicolson 1963) e centrifugação com sacarose (Daniels e Skipper 1982) a 60\% para extração e contagem dos glomerosporos. Foi realizada a diversidade dos FMA apenas para o período chuvoso. Para tal avaliaçáo, solos das leguminosas nas diferentes distâncias foram homogenizados. 
Os glomerosporos foram extraídos, separados por cor e tamanho e montados em lâmina permanentes com Polivinil Lacto-Glicerol (PVLG) e PVLG + Reagente de Melzer (Rossman 1980) para posterior identificação (Schenk e Perez 1990).

O potencial de micorrizaçáo (MIP) foi avaliado conforme bioensaio proposto por Brundrett (1991) onde amostras de solo sáo colocadas em potes e semeadas espécies de rápido crescimento radicular, tais como gramíneas, e, após um curto período de crescimento, as mesmas são colhidas e realizada a coloração das raízes para avaliar taxa de colonização micorrízica. Dois bioensaios foram realizados, um em cada época amostral, utilizando-se sorgo (Sorghum bicolor L.) no solo coletado das leguminosas (profundidade $0-20 \mathrm{~cm}$ e duas distâncias $0 \mathrm{~m}$ e $0,5 \mathrm{~m}$ ) e uma testemunha (fora do sistema em aléias), com quatro repetiçóes cada. Em casa de vegetação, vasos com capacidade de $500 \mathrm{~g}$ de solo foram semeados com cinco sementes e após cinco dias do plantio foi realizado o desbaste, deixando apenas uma planta por vaso. Após 40 dias de emergência do sorgo, avaliou-se: massa seca da parte aérea, taxa de colonizaçáo das raízes (Giovanetti e Mosse 1980) e o teor de P na parte aérea dos tecidos (Tedesco et al 1995).

Os dados foram submetidos ao teste de Bartlet e Lilliefor's e os que apresentaram $p>0,05$ sem distribuição normal foram transformados (número de glomerosporos/log $\mathrm{x}+1$ e taxa de colonização por arc sen X). Realizou-se análise de variância Two-way e para os tratamentos cujo teste $\mathrm{F}$ foi significativo, compararam-se as médias pelo teste de Tukey, ao nível de 5\% de significância. O programa usado para análise estatística foi o SAEG 8.0 (UFV 1997).

\section{RESULTADOS E DISCUSSÃO}

O potencial de infectividade de FMA nativos no cultivo em aléias variou de acordo com a leguminosa amostrada (Tabela 1). Menor número de glomerosporos foi encontrado nas amostras de solo coletadas na testemunha (cerca de 70 glomerosporos. $100 \mathrm{~g}$ de solo $^{-1}$ ) diferindo das leguminosas (média de 183 glomerosporos.100 $\mathrm{g} \mathrm{de} \mathrm{solo}^{-1}$ ). A maior densidade de glomerosporos foi encontrada no solo próximo a leucena e sombreiro (cerca de 200 glomerosporos. $100 \mathrm{~g}$ de solo $^{-1}$ ). Os solos sob influencia das leguminosas estimularam a colonização radicular do sorgo que foi superior a $50 \%$, contra $35,5 \%$ da testemunha.

A leucena e o sombreiro devem favorecer a multiplicação dos FMA por produzir mais raízes, principalmente, nos primeiros $30 \mathrm{~cm}$ de profundidade, sendo mais de $70 \%$ de raízes finas (Ferraz Júnior et al. 2006).

Linderman (1992), ao comparar um sistema de cultivo tradicional e um sistema em aléias, verificou que o número de glomerosporos era superior nas amostras oriundas das aléias, sugerindo que este sistema fosse benéfico para a manutenção das espécies de FMA já que as leguminosas permanecem na área nos sucessivos ciclos das culturas principais. Além disso, as podas realizadas rotineiramente podem promover a esporulação dos fungos, garantindo assim propágulos infectivos. Essa hipótese vem sendo levantada já que a planta sofre um estresse e o fungo recebe esses sinais químicos que podem promover esporulação.

Tabela 1 - Densidade de glomerosporos de fungos micorrízicos arbusculares (FMA) em sistema em aléias no Maranhão e taxa de colonização micorrízica (\%) nas raízes de sorgo de bioensaio semeado em solo de leguminosas do sistema em aléias e testemunha.

\begin{tabular}{lcc}
\hline Tratamentos & $\begin{array}{c}\text { Glomerosporos. } 100 \mathrm{~g} \text { de } \\
\text { solo }^{-1}\end{array}$ & Colonização (\%) \\
\hline Acácia & $127 \mathrm{~B}$ & $50,5 \mathrm{~A}$ \\
Leucena & $216 \mathrm{~A}$ & $52,7 \mathrm{~A}$ \\
Sombreiro & $225 \mathrm{~A}$ & $52,9 \mathrm{~A}$ \\
Testemunha & $73 \mathrm{C}$ & $35,5 \mathrm{~B}$ \\
\hline
\end{tabular}

Médias seguidas pela mesma letra na coluna não diferem significativamente pelo teste de Tukey a $5 \%$ de probabilidade.

A densidade de glomerosporos é menor no solo da testemunha (Figura 1), não apresentando efeito de época ( $\mathrm{p}$ $>0,05)$. Sob influência das leguminosas foi verificado efeito de época $(\mathrm{p}<0,05)$. De modo geral, registrou-se tendência de maior número de glomerosporos no período das chuvas (151 a 335 glomerosporos $100 \mathrm{~g}^{-1}$ de solo). A distância de coleta das amostras, em relação ao tronco da espécie vegetal também influenciou o número de glomerosporos, predominando maior número de propágulos nas amostras coletadas a $0 \mathrm{~m}$ do tronco das leguminosas.

$\mathrm{O}$ aumento do número de glomerosporos na estação chuvosa quando comparadas à estação seca também foi verificado por Silva et al. (2006). Entretanto, segundo Caproni et al (2005), o número de glomerosporos tende a diminuir com as chuvas, já que estes são estruturas de resistência e outras estruturas tais como as hifas seriam mais abundantes. De acordo com Guadarrama e Alvarez-Sánchez (1999), a umidade dos solos favorece a germinaçáo dos glomerosporos, reduzindo a produção destes e aumentando a de hifas. Allen et al. (1998), trabalhando com dinâmica sazonal de FMA em floresta tropical decídua, observaram que a colonização e esporulação eram maiores durante a estação chuvosa do que na seca, devido à maior atividade micorrízica naquele período, em consequência da maior quantidade de raízes na camada superficial do solo.

A diversidade de fungos micorrízicos da área incluiu 16 espécies de FMA distribuídas nos gêneros: Acaulospora, Ambispora, Scutellospora, Glomus e Gigapora, sendo elas: Acaulospora scrobiculata Trappe, A. morrowiae Spain \& N.C Schenck, A. tuberculata Janos \& Trappe, Ambispora appendicula (N.C. Schenck \& G.S Sm.) C.Walker, Vestberg \& 


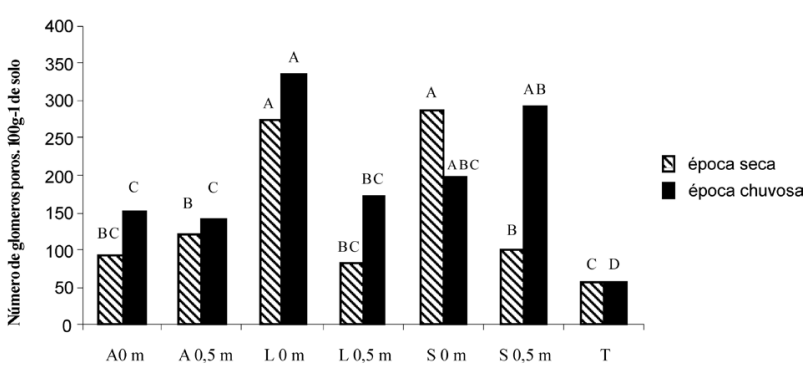

Figura 1 - Distribuição sazonal da densidade de glomerosporos de fungos micorrízicos arbusculares (FMA) em sistema em aléias e área testemunha ( $A=$ acácia, $L=$ leucena, $S=$ sombreiro e $T=$ testemunha), a diferentes distâncias das árvores $(0,0 \mathrm{~m}$ e 0,5 m). Barras encimadas pela mesma letra maiúscula comparam as leguminosas dentro das estações e minúsculas, entre as estações, não diferem estatisticamente pelo teste de Tukey a 5\% de probabilidade $(p>0,05)$

A. Schüßler, Scutellospora gregaria (N.C. Schenck \& G.S. Sm.) C. Walker, F.E. Sanders, S. castanea C. Walker, S. cerradensis Spain \& J. Miranda, S. calospora (T.H. Nicolson \& Gerd.) C. Walker \& F.E. Sanders, S. pellucida (T.H. Nicolson \& Gerd.) C. Walker \& F.E. Sanders), S. heterograma (T.H. Nicolson \& Gerd.) C. Walker \& F.E. Sanders, S. aurigloba (I.R. Hall) C. Walker \& F.E. Sanders, Scutellospora sp., Gigaspora decipiens I.R. Hall \& L.K. Abbott, G. albida (N.C. Schenck \& G.S. Sm.), Gigaspora sp. e Glomus sp.

A ocorrência destas espécies pode estar condicionada a variáveis edáficas como $\mathrm{pH}$ e disponibilidade de $\mathrm{P}$, Ca e $\mathrm{Mg}$, os quais determinam sua ocorrência, germinação e colonização de plantas (Siqueira et al. 1989, 1990). Entretanto, a determinação de correlaçôes entre ocorrência de FMA e estes fatores não é clara. $\mathrm{O}$ que se observa nos trabalhos de levantamento são tendências de ocorrências de FMA em função de algumas variáveis do solo. Sonjak et al.(2009) trabalhando em regiấo de solos com alta salinidade, verificaram a ocorrência de seis diferentes espécies de FMA, em sua maioria pertencentes ao gênero Glomus. Caproni $e t$ al (2007) observaram o predomínio de Acaulospora seguido de Glomus em solos contaminados com resíduo de mineração de bauxita no Pará.

Sabe-se que o pH afeta a distribuição e a abundância das diferentes espécies de fungos micorrízicos (Wang et al. 1993). Desse modo, pequenos aumentos no pH estáo associados a grandes taxas de colonização em solos ácidos e com baixos teores de P disponível (Heijne et al. 1996).

$\mathrm{O}$ solo estudado ( $\mathrm{pH}$ entre 4,0 - 4,4 e teores de $\mathrm{P}$ entre 6 a $17 \mathrm{mg} \cdot \mathrm{dm}^{-3}$ ) apresentou apenas uma espécie de Glomus, três de Gigaspora e Acaulospora. Verifica-se que o gênero mais frequente foi Scutellospora (oito espécies), o que permite supor que há favorecimento desse gênero no ambiente estudado (solos ácidos). Entretanto, esses resultados não confirmam os de Siqueira et al. (1989) que observaram a tendência de
Acaulospora ocorrer com maior frequência em solos com pH menor que 6,5 enquanto Gigaspora eram menos frequentes. Espécies de Glomus mostraram-se pouco tolerantes à acidez elevada e baixo teor de fósforo. Contudo, esses resultados devem ser analisados com cuidado já que foram montadas lâminas apenas da estação chuvosa, onde pode haver maior influencia das mudanças sazonais e estádio de desenvolvimento da planta hospedeira nas espécies que estão esporulando no momento da coleta (Giovannetti 1985; Gould et al. 1996; Guadarrama e Alvarez-Sânchez 1999, Caproni et al.2007). Amostragens sazonais e utilização de culturas armadilhas devem ser realizadas para melhor estimativa da diversidade de FMA. Além disso, o uso de técnicas moleculares pode ser ferramenta interessante para tal quantificação.

No bioensaio, verificando o parâmetro distância, observase que o sorgo semeado em solo da acácia a $0 \mathrm{~m}$ acumulou menos massa seca da parte aérea (MSPA) enquanto o sombreiro a $0,5 \mathrm{~m}$ apresentou menor valor quando comparado ao sombreiro a $0 \mathrm{~m}$ (Tabela 2 ).

Tabela 2 - Efeito das interações do bioensaio com plantas de sorgo semeadas em solo de leguminosas do sistema em aléias e distâncias amostradas $(0 \mathrm{~m}$ e $0,5 \mathrm{~m}$ ) na colonização micorrízica, massa seca da parte aérea (MSPA) e teor de fósforo na parte aérea do sorgo.

\begin{tabular}{lcccc}
\hline Leguminosas & Distância $(\mathrm{m})$ & Colonização \% & MSPA $(\mathrm{g})$ & $\begin{array}{c}\text { Teor de P } \\
\left(\mathrm{g} \cdot \mathrm{kg}^{-1}\right)\end{array}$ \\
\hline Acácia & & $50,8 \mathrm{~ns}$ & $0,065 \mathrm{Ba}$ & $6,89 \mathrm{AB}$ \\
Leucena & 0,0 & 52,2 & $0,079 \mathrm{Aba}$ & $6,51 \mathrm{Aa}$ \\
Sombreiro & & 54,1 & $0,106 \mathrm{Aa}$ & $6,09 \mathrm{Aa}$ \\
Acácia & & 54,0 & $0,068 \mathrm{Aa}$ & $9,47 \mathrm{Aa}$ \\
Leucena & 0,5 & 53,4 & $0,085 \mathrm{Aa}$ & $4,56 \mathrm{Cb}$ \\
Sombreiro & & 55,2 & $0,062 \mathrm{Ab}$ & $7,42 \mathrm{Ba}$ \\
\hline
\end{tabular}

Médias seguidas pela mesma letra maiúscula na coluna comparam dentro da distância e minúsculas, dentro da mesma espécie de leguminosa, não diferem estatisticamente pelo teste de Tukey a $5 \%$ de probabilidade. ns = não significativo $(p>0,05)$.

Não houve diferença entre as espécies de leguminosas dentro da mesma estaçáo do ano, no entanto, o sorgo cresceu mais em solo coletado na estação chuvosa (Tabela 3). Os teores de $\mathrm{P}$ na parte aérea do sorgo do bioensaio foram mais elevados nos solos das leguminosas a $0,5 \mathrm{~m}$, exceto para a leucena (Tabela 2). Também houve efeito da época de coleta do solo, onde o sorgo semeado no solo da época chuvosa apresentou maiores teores de P nos tecidos (Tabela 3). Quanto à colonizaçâo radicular do sorgo, verificou-se que as épocas de amostragem foram mais determinantes que as distâncias, sendo o período chuvoso a época com maiores índices de colonização radicular do bioensaio (Tabelas 2 e 3 ).

Resultados de colonização com o bioensaio (Tabelas 1, 2 e 3) foram superiores aos obtidos por Diagne et al.(2001) quando obtiveram valores baixos, variando entre $5 \%$ e $32 \%$ de infecção radicular. Esses resultados de densidade de glomerosporos e taxa de colonização indicam que o sistema em 
aléias favorece o potencial de micorrizaçáo dos FMA nativos quando comparados ao sistema convencional de cultivo.

Tabela 3 - Efeito das interações do bioensaio com plantas de sorgo semeado em solo de leguminosas do sistema em aléias e estação do ano (seca e chuvosa) para a colonização micorrízica, massa seca da parte aérea (MSPA) e teor de fósforo na parte aérea do sorgo.

\begin{tabular}{lcccc}
\hline Leguminosas & Estação & Colonização \% & MSPA (g) & $\begin{array}{c}\text { Teor de P } \\
\left(\mathrm{g} . \mathrm{kg}^{-1}\right)\end{array}$ \\
\hline Acácia & & $55,4 \mathrm{Aa}$ & $0,048 \mathrm{Ab}$ & $6,70 \mathrm{Ab}$ \\
Leucena & seca & $46,3 \mathrm{Bb}$ & $0,059 \mathrm{Ab}$ & $3,88 \mathrm{Bb}$ \\
Sombreiro & & $55,7 \mathrm{Aa}$ & $0,051 \mathrm{Ab}$ & $3,26 \mathrm{Bb}$ \\
Acácia & & $45,7 \mathrm{Cb}$ & $0,085 \mathrm{Aa}$ & $9,66 \mathrm{Aa}$ \\
Leucena & chuvosa & $59,1 \mathrm{Aa}$ & $0,105 \mathrm{Aa}$ & $10,2 \mathrm{Aa}$ \\
Sombreiro & & $50,3 \mathrm{Bb}$ & $0,117 \mathrm{Aa}$ & $7,18 \mathrm{Ba}$ \\
\hline
\end{tabular}

Médias seguidas pela mesma letra na coluna maiúscula comparam dentro da estação e minúsculas, dentro da mesma espécie de leguminosa, não diferem estatisticamente pelo teste de Tukey a $5 \%$ de probabilidade.

\section{CONCLUSÕES}

O sistema em aléias estudado aumenta o potencial de infectividade dos FMA nativos, sendo que este varia com a espécie de leguminosa arbórea associada, estação chuvosa e as proximidades das árvores.

O gênero predominante de fungos micorrízicos durante o período chuvoso foi Scutellospora, demonstrando melhor adaptação aos solos ácidos da área em estudo.

A quantidade de glomerosporos não possui relaçáo direta com a taxa de colonizaçáo das raízes de sorgo e capacidade infectiva dos propágulos dos fungos micorrízicos.

\section{BIBLIOGRAFIA CITADA}

Allen, E.B.; Rincon, E.; Allen, M.F.; Jimenez, A.P.; Huante, P. 1998. Disturbance and seasonal dynamics of mycorrhizae in a tropical deciduous forest in México. Biotropica, 30: 261-274.

Bala, A.; Murphy, P.; Guiller, K.E. 2003. Distribution and diversity of rhizobia nodulating agroforestry legumes in soil from three continents in the tropics. Molecular Ecology, 12: 917-930.

Bonfim, J.A.; Matsumoto, S.N.; Lima, J.M.; César, F.R.C.F.; Santos, M.A.F. 2010. Arbuscular Mycorrhizal Fungi and Physiological Aspects of Coffee Conducted in Agroflorestal System and at full Sun. Bragantia, 69(1): 201-206 (in Portuguese with abstract in English).

Brundrett, M.C. 1991.Mycorrhizas in natural ecosystems. In: Macfayden, A. Begon, M. Fitter, A.H. (eds.), Advances in Ecological Research. Academic Press, London, 99:171-313.

Buresh, R.J.; Tian, G. 1998. Soil improvement by trees in subSaharan Africa. Agroforestry Systems, 38: 51-76.

Caproni, A.L.; Franco, A.A.; Berbara,R.L.L.; Granha, J.R.D.O.; Marinho, N.F. 2005. Colonization of arbuscular mycorrhizae fungi in substrate, after bauxite mining, vegetated with Acacia mangium. Revista Arvore, 29(03): 373-381 (in Portuguese with abstract in English).
Caproni,A.L.; Franco, A.A.; Granha, J.R.D.O.; Souchie, E.L. 2007. Arbuscular mycorrhizal fungi occurrence in bauxite mining residue planted to tree species. Acta Botanica Brasilica 21(1): 99-106. (in Portuguese with abstract in English).

Cardoso, I.M.; Boddington,C.; Janssen,B.H.; Oenema, O.; Kuyper, T.W. 2003. Distribution of mycorrhizal fungal spores in soils under agroforestry and monocultural coffee systems in Brazil. Agroforestry Systems, 58: 33-43.

Cardoso, I.M.; Kuyper, T.M. 2006. Mycorrhizal and tropical soil fertility agriculture. Agriculture Ecosystems \& Environment. 116: 72-84.

Coopper, P.J.M.; Leakey, R.R.B.; Rao, M.R.; Reynolds, L. 1996. Agroforestry and the mitigation of land degradation in the humid and sub-humid tropics of Africa. Experimental Agriculture, 32: 235-290.

Daniels, B.A.; Skipper, H.D. 1982. Methods for the recovery and quantitative estimation of propagules from soil. pp. 20-45 In: N. C. Schenck (ed.), Methods and principles of mycorrhizal research, The American Phytopathological Society, St. Paul.

Diagne, O.; Ingleby, K.; Deans, J.D.; Lindley, D.K.,; Diaité, I.; Neyra, M. 2001. Mycorrhizal inoculum potencial of soils from alley cropping plots in Sénégal. Forest Ecology and management, 146: 35-43.

Ferraz Júnior, A.S.de L. 2006. The alley cropping system as na alternative to food producticion of food in a smalholder system in the Humid Tropic. In: (org.) Moura, E. G. Transitions Agrossistems:from the humid tropics and semi-arid region of Brazil. Série Agroecologia - São Luis: UEMA. vol.I, 2.edição. (in portuguese)

Ferraz Júnior, A.S.L.; Souza, S.R.; Satrk, E.M.L.; Fernandes,M.S.2006. Phytomass, Root Distribution and Input of Nitrogen and Phosforus by Leguminous in Alley Cropping in Low Fertility Soil. Revista Floresta e Ambiente: UFRRJ. 13 (1): 61-68 (in Portuguese with abstract in English).

Gerdemann, J. W.; Nicolson, T.H. 1963. Spores of mycorrhizal Endogone species extracted from soil by wet-sieving and decanting. Transactions of the British Mycological Society. 46: 235-244.

Giovanetti, M.; Mosse, B. 1980. An evaluation of techniques of measuring vesicular arbuscular mycorrhizal infection in roots. New Phytologist, 84: 484-500.

Giovannetti, M. 1985. Seasonal variations of vesicular arbuscular mycorrhizas and endogonaceous spores in a maritime sand dune. Transactions of the British Mycological Society, 84: 679-684.

Gould, A.B.; Hendrix, J.W.; Ferriss, R.S. 1996. Relationship of mycorrhizal activity to time following reclamation of surface mine land in Western Kentucky - I: propagule and spore population densities. Canadian Journal of Botany, 74: 247-261.

Guadarrama, P.; Álvarez-Sánchez, F.J. 1999. Abundance of arbuscular mycorrhizal fungi spores in different environments in a tropical rain for Veracruz, Mexico. Mycorrhiza 8: 267- 270.

Heijine, B.; Van Dam, D.; Heil,G.W.; Bobbik, R. 1996. Acidification effects on vesicular-arbuscular mycorrhizal (VAM) infection, 
growth and nutrient uptake of established heathland herb species. Plant Soil, 179: 197-206.

Linderman, R.G. 1992. Vesicular-Arbuscular Micorrhyzae and Soil Micorbial interactions. In: Bethen Falvay, G.J.; Linderman, R.G. Mycorrhizal in Sustainable Agriculture. American Society of Agronomy, Madison - USA.

Nielsen, K.B.; Kjøller, R.; Olsson, P.A.; Schweiger, P.F.; Andersen, F.Ø.; Rosendahl, S. 2004. Colonization intensity and molecular diversity of arbuscular mycorrhizal fungi in the aquatic plants Littorella uniflora and Lobelia dortmanna in southern Sweden. Mycological Research, 108: 616-625.

Oyetunji,O.J.; Osonubi, O. 2007. Assessment of Influence of Alley Cropping System and Arbuscular Mycorrhizal (AM) Fungi on Cassava Productivity in Derived Savanna Zone of Nigeria. World Journal of Agricultural Sciences, 3 (4): 489-495.

Pande, M.; Tarafdar, J.C. 2004. Arbuscular mycorrhizal fungal diversity in neen-based agroforestry systemas in Rajasthan. Applied Soil Ecology. 26: 233-241.

Read, D.J. 1991. Mycorrhizas in ecosystems. Experientia, 47: 376-390.

Rossman, A.Y. 1980. The iodine reaction: Melzer's vs. IKI. MSA newsletter 31: 22.

Rowe, E.C.; Cadish, G. 2002. Implications of heterogeneity on procedures for estimating plant $\mathrm{N}_{15}$ recovery in hedgerow intercrop systems. Agroforestry Systems, 54: 61-70.

Sanchez, P.A. 1995. Science in Agroforestry. Agroforestry Systems 30: $5-55$.

Schenck,N.C.; Pérez, Y. 1990. Manual for the identification of VAmycorrhizal fungi. 3.ed. Gainesville: Synergistics - Publications, $250 \mathrm{p}$.

Schüßler A.; Schwarzott D,; Walker C. 2001. New fungal phylum, the Glomeromycota: phylogeny and evolution. Mycological Research, 105: 1413-1421.
Silva, C.F.; Pereira, M.G.; Silva, E.M.R.; Correia, M.E.F.; SagginJúnior, O.J. 2006. Mycorrhizal fungi in surrounding areas of Parque Estadual da Serra do Mar in Ubatuba (SP). Revista Caatinga (Mossoró, Brasil), 19 (1): 01-10. (in Portuguese with abstract in English).

Siqueira, J. O.; Rocha Júnior, W. F.; Oliveira, E.; Colozzi-Filho, A. 1990. The relationship between vesicular-arbuscular mycorrhiza and lime: associated effects on the growth and nutrition of Brachiaria grass (Brachiaria decumbens). Biology and Fertility of Soils, 10: 65-71.

Siqueira, J.O.; Colozzi-Filho, A.;Oliveira, E.; Schenck, N.C. 1989. Occurence of mycorrhizal fungi in agro and ecossystems in Minas Gerais State.. Pesquisa Agropecuária Brasileira, 24:1499-1506. (in Portuguese with abstract in English).

Sonjak, S.; Udovic, M.; Wraber, T.; Likar, Matevz.; Regvar, M. 2009. Diversity of halophytes and identification of arbuscular Mycorrhizal fungi colonizing their roots in an abandoned and sustained part of Secovlje salterns. Soil Biology \& Biochemistry, 41: 1847-1856.

Tedesco, M. J.; Gianello, C.; Bissani, C. A.; Bohnen, H.; Volkweiss, S. J.1995. Soil analisys, plants and other materials. 2. ed. Porto Alegre, Universidade Federal do Rio Grande do Sul, 174p. (Boletim técnico, 5). (in Portuguese)

Universidade Federal de Viçosa - UFV. Central de Processamento de Dados UFV/CPD. 1997. SAEG-System for statistic and genetics analisys. Versão 8.0. Viçosa, MG: UFV, 54p. (in Portuguese)

Wang,G.M.; Stribley,D.P.; Tinker, P.B.; Walker, C. 1993. Effects of $\mathrm{pH}$ on arbuscular mycorrhiza I. Field observations o the longterm liming experiments at Rothamsted and Wobum. New Phytologist, 124: 465-472.

Recebido em 28/01/2010

Aceito em 25/08/2010 